\title{
Magnetic Phase Transitions in TbNi(Al,In) Compounds
}

\author{
M. Klicpera ${ }^{a, *}$, P. JAVOrskÝ ${ }^{a}$ AND E. ŠAnTAVÁ ${ }^{b}$ \\ ${ }^{a}$ Charles University in Prague, Faculty of Mathematics and Physics, Department of Condensed Matter Physics \\ Ke Karlovu 5, 12116 Prague 2, Czech Republic \\ ${ }^{b}$ Institute of Physics ASCR, v.v.i., Na Slovance 2, 18221 Prague 8, Czech Republic
}

\begin{abstract}
The magnetic phase transitions in $\mathrm{TbNiAl}_{1-x} \mathrm{In}_{x}$ compounds were investigated by ac-susceptibility measurements. Our data reveal magnetic ordering with transition temperatures between 40 and $70 \mathrm{~K}$, depending on the In concentration. All the studied compounds exhibit two or more phase transitions accompanied by distinct anomalies in the real and imaginary part of the ac-susceptibility. The paramagnetic Curie temperatures remain positive in the whole series.
\end{abstract}

PACS numbers: 75.30.-m, 75.40.Gb

\section{Introduction}

The ternary RTX compounds $(\mathrm{R}=$ rare earth, $\mathrm{T}=$ transition $d$-metal, $\mathrm{X}=p$-metal) crystallizing in the hexagonal ZrNiAl-type structure (space group $P \overline{6} 2 m$ ) form a large group of intermetallics. Most of these compounds order magnetically at low temperatures, often with complex magnetic structures (see e.g. [1-3]). Generally, the type of magnetic order is very sensitive to the number of conduction electrons and the development of magnetic properties in pseudo ternary series like $\mathrm{R}(\mathrm{Ni}, \mathrm{Cu}) \mathrm{Al}$ is very complex [4]. On the other hand, magnetocrystalline anisotropy seems to be strongly influenced by the ratio of lattice parameters which is most noticeable in the Tb-based compounds showing the strongest anisotropy. To bring deeper information about the relation between magnetic anisotropy and crystal structure, the $\mathrm{TbNi}(\mathrm{Al}, \mathrm{In})$ series seems to be ideal. The Al-In substitution is isoelectronic, so the changes of magnetic structure are primarily driven by the large change of the lattice parameters that occurs in this series $[5,6]$. Both parent compounds have been previously studied. $\mathrm{TbNiAl}$ orders antiferromagnetically below $T_{\mathrm{N}}=47 \mathrm{~K}$ and undergoes further magnetic phase transition at $T_{1}=23 \mathrm{~K}$ [5]. One third of $\mathrm{Tb}$ moments is reduced to almost zero between $T_{\mathrm{N}}$ and $T_{1}$, change of the propagation of the frustrated moments occurs below $T_{1}$. The $\mathrm{Tb}$ magnetic moments are oriented parallel to the $c$-axis in both magnetic phases [5]. TbNiIn orders magnetically below $T_{\text {ord }} \approx 70 \mathrm{~K}$ with additional magnetic phase transitions indicated by ac-susceptibility at $T_{1}=59 \mathrm{~K}$ and $T_{2}=29 \mathrm{~K}[6]$. As inferred from neutron diffraction, the $\mathrm{Tb}$ moments lie within the basal plane

\footnotetext{
* corresponding author; e-mail: Mi.Klicpera@seznam.cz
}

and form a non-collinear structure below the transition temperature $T_{\text {ord }}$, while an additional weaker component develops below $T_{2}$ [7]. We give a basic characterization of the magnetic properties of $\mathrm{TbNi}(\mathrm{Al}$,In) compounds in this paper.

\section{Experimental}

Polycrystalline $\mathrm{TbNiAl}_{1-x} \mathrm{In}_{x}$ samples were prepared by arc-melting of pure elements in a mono-arc furnace under the protection of an argon atmosphere. Small excess of In was used $(\approx 5 \%)$ to compensate higher evaporation of In during the melting. The X-ray diffraction patterns obtained using $\mathrm{Cu} K_{\alpha}$ radiation confined single-phase samples within $\mathrm{ZrNiAl}$-type of structure and the energy dispersive X-ray (EDX) analysis confirmed homogeneous $\mathrm{In}-\mathrm{Al}$ distribution. To determine the paramagnetic characteristics, magnetization was measured between 50 and $300 \mathrm{~K}$ in the field of $2 \mathrm{~T}$. The temperature variation of the ac-susceptibility was measured during cooling between 80 and $3 \mathrm{~K}$ using the PPMS (Quantum Design) instrument. The data in $0 \mathrm{~T}$ were obtained for frequencies 13 , 113, 1113 and $9993 \mathrm{~Hz}$, the frequency of $9993 \mathrm{~Hz}$ was additionally used for measurements in magnetic fields of $0.05,0.10,0.20$ and $0.50 \mathrm{~T}$. Both magnetization and ac-susceptibility were measured on powder samples randomly fixed by a glue.

\section{Results and discussion}

In the paramagnetic region, the $M / H(T)$ dependences follow the Curie-Weiss behavior. The determined values of the effective moment, $\mu_{\mathrm{eff}}$, are close or slightly higher than the $\mathrm{Tb}^{3+}$ free ion value of $9.72 \mu_{\mathrm{B}}$ (see Table). The small enhancement can be attributed to polarization of conduction electrons, similar to pure TbNiAl [5]. The 
paramagnetic Curie temperature, $\theta_{\mathrm{p}}$, is positive for all In concentrations and weakly increases for the In rich compounds. The positive $\theta_{\mathrm{p}}$ in $\mathrm{TbNiAl}$ despite its antiferromagnetic structure points to prevailed ferromagnetic interactions in the paramagnetic region [5].

TABLE

Magnetic phase transition temperatures as determined from ac-susceptibility and the paramagnetic characteristics (not measured for $x=0.0,0.3,0.7$ and 1.0) in $\mathrm{TbNiAl}_{1-x} \mathrm{In}_{x}$ compounds.

\begin{tabular}{l|c|c|c|c|c}
\hline \hline \multicolumn{1}{c|}{$x$} & $\begin{array}{c}T_{\text {ord }} \\
{[\mathrm{K}]}\end{array}$ & $\begin{array}{c}T_{1} \\
{[\mathrm{~K}]}\end{array}$ & $\begin{array}{c}T_{2} \\
{[\mathrm{~K}]}\end{array}$ & $\begin{array}{c}\mu_{\text {eff }} \\
{\left[\mu_{\mathrm{B}}\right]}\end{array}$ & $\begin{array}{c}\theta_{\mathrm{P}} \\
{[\mathrm{K}]}\end{array}$ \\
\hline $0.0[5]$ & 47 & 23 & - & 10.4 & 30 \\
0.0 & 46 & 24 & 18 & & \\
0.1 & 48 & 25 & 18 & 9.9 & 36 \\
0.2 & 46 & 22 & - & 10.0 & 32 \\
0.3 & 45 & 15 & - & & \\
0.4 & 42 & 12 & - & 10.1 & 34 \\
0.5 & 42 & 12 & - & 9.6 & 34 \\
0.6 & 45 & 14 & - & 9.9 & 36 \\
0.7 & 47 & 20 & - & & \\
0.8 & 51 & 20 & 13 & 9.7 & 40 \\
0.9 & 60 & 49 & 21 & 9.6 & 44 \\
1.0 & 72 & 54 & 20 & & \\
$1.0[6]$ & 70 & 59 & 29 & 9.5 & 55
\end{tabular}

The temperature dependence of ac-susceptibility reveals two or more phase transitions for all studied compounds. The anomalies related to the temperature of magnetic ordering are frequency independent which indicates the long-range magnetic order in the whole series, contrary to some $\mathrm{R}(\mathrm{Ni}, \mathrm{Cu}) \mathrm{Al}$ compounds where the spin-glass state is manifested by frequency dependent signal [8]. The ac-susceptibility of $\mathrm{Al}$ rich compounds show qualitatively similar behaviour to the parent $\mathrm{TbNiAl}$ with a relatively sharp anomaly at $T_{\mathrm{N}}$ and less pronounced maximum around $T_{1}$ (see Fig. 1). With increasing the In content, the anomaly around the ordering temperature becomes broader and the low-temperature anomaly becomes more pronounced, especially in $\chi^{\prime \prime}$. Two maxima are observed at the low-temperature region at 20 and $13 \mathrm{~K}$ for $x=0.8$, indicating more complex behaviour. The main maximum in the In rich compounds $\mathrm{TbNiAl}_{0.1} \mathrm{In}_{0.9}$ and $\mathrm{TbNiIn}$ is preceded by certain shoulder at higher temperatures (see Fig. 2), which was observed also in the previous study on TbNiIn [6]. All the observed anomalies are summarized in Table. The magnetic field generally suppresses all the anomalies but causes also a shift of some of them to lower temperatures which could indicate the antiferromagnetic character (see Figs. 1 and 2).

We can conclude that $\mathrm{TbNi}(\mathrm{Al}, \mathrm{In})$ compounds order magnetically with transition temperatures between 40 and $70 \mathrm{~K}$, and show existence of at least two different magnetic phases. To investigate the magnetic structures

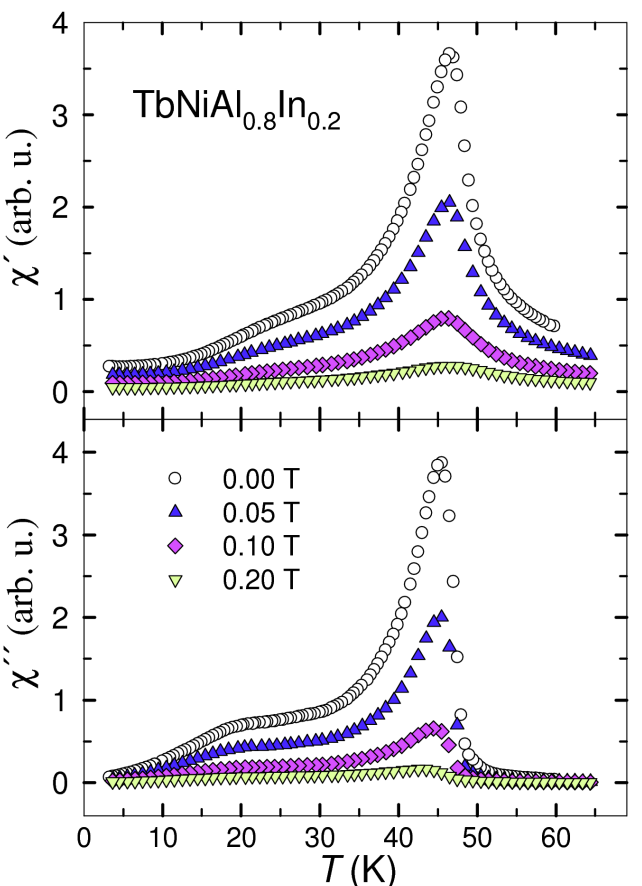

Fig. 1. Ac-susceptibility (real (a) and imaginary (b)) of $\mathrm{TbNiAl}_{0.8} \mathrm{In}_{0.2}$ measured in different magnetic fields. The data obtained for $0 \mathrm{~T}$ were divided by a factor of $5 \times 10^{4}$ for better lucidity.

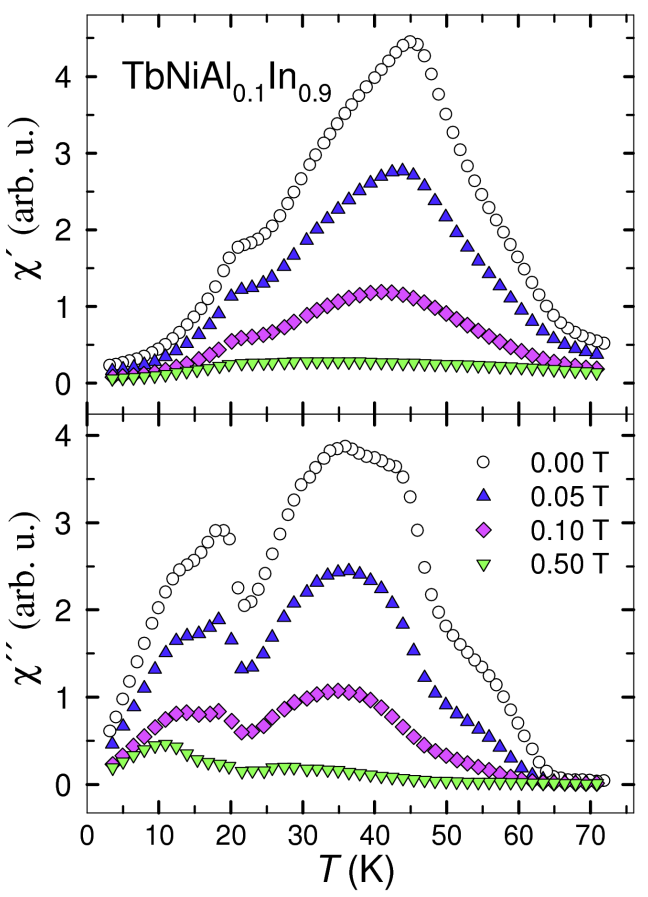

Fig. 2. Ac-susceptibility (real (a) and imaginary (b)) of $\mathrm{TbNiAl}_{0.1} \mathrm{In}_{0.9}$ measured in different magnetic fields. The data obtained for $0 \mathrm{~T}$ were divided by a factor of $3 \times 10^{4}$ and the $0.5 \mathrm{~T}$ data were multiplied by a factor of 4 for better lucidity. 
of individual phases, neutron diffraction study will be performed.

\section{Acknowledgments}

The work was supported by the Grant Agency of the Czech Republic under the grant no. 202/08/0711, the work of M.K. was supported by the grant SVV-2010-261303. This work is a part of the research program MSM 0021620834 financed by the Ministry of Education of the Czech Republic.

\section{References}

[1] G. Ehlers, H. Maletta, Z. Phys. B 101, 317 (1996).

[2] O. Garlea, E. Morosan, S.L. Bud'ko, J.L. Zarestky, P.C. Canfield, C. Stassis, J. Appl. Phys. 95, 6921 (2004).
[3] S. Baran, A. Arulraj, D. Kaczorowski, B. Penc, A. Szytuła, Intermetallics 18, 42 (2010).

[4] J. Prchal, T. Naka, M. Míšek, O. Isnard, P. Javorský, J. Magn. Magn. Mater. 316, e499 (2007).

[5] G. Ehlers, H. Maletta, Z. Phys. B 99, 145 (1996).

[6] Yu.B. Tyvanchuk, Ya.M. Kalyczak, Ł. Gondek, M. Rams, A. Szytuła, Z. Tomkowicz, J. Magn. Magn. Mater. 277, 368 (2004).

[7] L. Gondek, A. Szytula, S. Baran, J. Hernandez-Velasco, J. Magn. Magn. Mater. 272-276, e443 (2004).

[8] J. Prchal, E. Šantavá, D. Schmoranzer, Physica B 404, 3056 (2009). 